\title{
Acute corticosteroid-induced rhabdomyolysis in a golf player
}

\author{
Section Sports Medicine, University of Pretoria \\ D C Janse van Rensburg, MD \\ T C Grant, BSc \\ P Cele Zondi, $\mathrm{MB} \mathrm{ChB}$ \\ C C Grant, PhD
}

D C Janse van Rensburg, W Theron, T C Grant, P Cele Zondi, C C Grant

Physician in private practice (collaborating with Section Sports Medicine)

W Theron, FCP (SA)

Corresponding author: D C Jansevan Rensburg (christa.jansevanrensburg@up.ac.za)

Acute corticosteroid-induced rhabdomyolysis is a rare, but potentially life-threatening, condition that deserves the attention of medical professionals and sport scientists. Early diagnosis is vital in minimising the secondary damage caused by rhabdomyolysis. This case of rhabdomyolysis highlights the severity of symptoms and the importance of decisive treatment. Clinicians should be familiar with the most common symptoms of acute corticosteroid-induced rhabdomyolysis to enable early diagnosis and efficient management of this condition.

S Afr J SM 2012;24(4):129-130. DOI:10.7196/SAJSM.339

The simultaneous destruction of skeletal muscle cells with the consequent release of cellular contents into the circulatory system is called rhabdomyolysis and can be triggered by mechanical injury, ischaemia, infections, or genetic alterations to drugs and toxins. ${ }^{1}$ The intrinsic characteristics of muscle tissue make it especially vulnerable to drug-related cell damage. Although rare, the life-threatening illness of acute steroid myopathy causing rhabdomyolysis in patients on high-dose corticosteroids should be recognised by clinicians and sports medicine specialists.

This article presents the case of a young golf player with rhabdomyolysis, as well as current theories on cellular mechanisms, and symptoms and treatment of acute corticosteroid-induced rhabdomyolysis.

\section{Case report}

A 27-year-old professional golf player presented with neck and back pain suggestive of an inflammatory arthropathy. Symptoms included polyarthralgia of the elbows, shoulders, knees and right foot as well as morning stiffness for longer than one hour. The morning stiffness and polyarthritis responded to anti-inflammatory drugs.

On physical examination he had a normal Schober test and chest expansion. While systemic and neurological examination was normal, the FABER test was positive bilaterally with tenderness of the sacroiliac (SI) joints and thoracic spinous processes. The initial differential diagnosis included possible spondylarthropathy, and mechanical neck and back pain.

Further tests revealed a normal full blood count. Erythrocyte sedimentation rate was $2 \mathrm{~mm} / \mathrm{h}$ and C-reactive protein levels were $2 \mathrm{mg} / \mathrm{l}$. HLA B27, rheumatoid factor and anti-cyclical citrillunated peptide antibody tests were negative. The isotope bone scan reported low-grade inflammatory changes in the superior part of the SI joints and normal uptake in the spine and peripheral joints. The lumbar spine radiograph showed slight degeneration of the L5/S1 facet joints.
The neck radiograph showed torticollis, while the thoracic spine radiograph showed kyphosis.

Having failed to respond to physiotherapy and anti-inflammatory drugs, he was admitted to hospital for a trial of intravenous therapeutic agents. A high dose of intravenous cortisone (125 mg methylprednisolone twice daily) was infused, backache of inflammatory origin typically responding to this form of management. The baseline creatine kinase (CK) level was 67 IU/l. The following day (day 2 of hospital admission) the patient had generalised myalgia and stiffness. CK levels were elevated at 1568 IU/l and myoglobin at $448 \mathrm{mg} / \mathrm{ml}$. Phosphate, magnesium and electrolyte levels were normal, as was troponin T. Thyroid function was also normal and the patient's history and clinical course were not supportive of metabolic myopathy. The patient also denied any changes in his training programme, the use of drugs such as statins or colchisine, nutritional supplementation or exposure to toxins such as snake venom. Therefore all other possible causes for the elevated CK and myoglobin levels were eliminated.

On day 3 of hospital admission, the patient's CK was markedly elevated at $4609 \mathrm{IU} / \mathrm{l}$ and the myoglobin was $1566 \mathrm{ng} / \mathrm{ml}$. He was treated with intravenous fluid, sodium bicarbonate and mannitol to prevent renal failure and arrhythmias due to increased potassium levels. The cortisone treatment was also discontinued. On the fourth day of hospitalisation the CK level was $8030 \mathrm{IU} / \mathrm{l}$ and the myoglobin $172 \mathrm{mg} / \mathrm{ml}$. On day 5 the levels decreased to $3301 \mathrm{IU} / \mathrm{l}$ and $129 \mathrm{mg} / \mathrm{ml}$, respectively (the second day after cortisone treatment was stopped).

The diagnosis of acute steroid-induced rhabdomyolysis was based on the combination of symptoms and biochemical markers with subsequent clearance of both after cessation of steroid therapy.

\section{Discussion}

The association between high-dose corticosteroid administration and muscle weakness was first reported by Cushing in $1932 .{ }^{2}$ 
Corticosteroid-induced myopathy is a disease that mainly causes weakness of the proximal muscles of the upper and lower limbs and the neck flexors. ${ }^{3}$ It is caused by an excess of corticosteroids from endogenous or exogenous sources such as adrenal tumours or steroid treatment. There are two distinct types of corticosteroidinduced myopathy, namely chronic and acute, ${ }^{3}$ which may pose the risk of rhabdomyolysis development. The chronic type of myopathy is caused by prolonged exposure to corticosteroids, while the acute type is less common and occurs abruptly when the patient is receiving high doses of corticosteroids. Previous cases of myopathy have been reported in patients receiving cortisone treatment for acute asthma, ${ }^{2}$ patients with phaeochromocytoma, ${ }^{4}$ and those in intensive care units. ${ }^{5}$

Acute myopathy induced by high-dose corticosteroid treatment differs significantly from chronic myopathy. This suggests a different pathogenesis. ${ }^{2}$ Willams et al. ${ }^{2}$ described the main differences between chronic and acute myopathy as listed in Table 1.

\section{Cellular mechanism of rhabdomyolysis}

The specific physiological mechanisms by which the different drugs act during rhabdomyolysis are uncertain. ${ }^{3,6}$ Current research suggests that several factors play a role in the development of rhabdomyolysis, such as decreased protein synthesis and sarcolemmal excitability, increased protein degradation, modifications in carbohydrate metabolism, mitochondrial changes and electrolyte disturbances. Steve Lim (2010) suggests that the use of fluorinated steroids such as dexamethasone or triamcinolone more frequently causes steroid myopathy than non-fluorinated steroids such as prednisone or hydrocortisone. ${ }^{3}$ Muscles that are less active are more likely to be affected by corticosteroid, meaning that a sedentary lifestyle may increase the risk of muscle weakness. ${ }^{3,5}$ Interestingly, gender also seems to be a risk, as women are twice as likely as men to develop muscle weakness. ${ }^{4}$

Martin Hohenegger ${ }^{6}$ described the cellular mechanism by which rhabdomyolysis develops. It begins with a decrease in intracellular ATP and a simultaneous increase in myoplasmic calcium $\left(\mathrm{Ca}^{2+}\right)$ concentrations. ${ }^{6}$ This leads to a deficiency in ATP supplied by the mitochondrial chain, leading to reduced replenishment of $\mathrm{Ca}^{2+}$ stores and extrusion of $\mathrm{Ca}^{2+}$ to the extracellular space. ${ }^{6}$ Calpain proteases are activated by the long-term elevated $\mathrm{Ca}^{2+}$ levels that further degrade the proteins participating in $\mathrm{Ca}^{2+}$ homeostasis. This intensifies the myoplasmic $\mathrm{Ca}^{2+}$ overload. ${ }^{7}$ High cytosolic calcium has various detrimental effects. Firstly, the mitochondria are overcome, leading to the disruption of oxidative phosphorylation, which decreases the available ATP. Secondly, apoptosis is triggered by the increased production of reactive oxygen species by the mitochondria, leading to the free radical disruption of cell and organelle membranes. Thirdly, a series of proteases and phospholipases are activated, damaging the myofibrillar network. ${ }^{1}$

\section{Symptoms and treatment}

Rhabdomyolysis is preceded by muscle weakness, muscle stiffness, symptoms of myalgia, swelling, tenderness and tea-coloured urine. ${ }^{1}$ There are, however, no clear laboratory parameters that estimate the risk a patient has of developing rhabdomyolysis, ${ }^{6}$ and a single symptom is not enough to make a definite diagnosis of rhabdomyolysis. Clinicians should be alert to the presence of the following possible indicators: ${ }^{1}$

- A serum CK level $>1000 \mathrm{U} / 1$ is important in determining whether the patient is suffering from rhabdomyolysis. ${ }^{1}$ Typically, the serum concentration of CK rises in the first 12 hours after injury, peaks at 3 days, and normalises at approximately 5 days. ${ }^{1}$

- Damaged muscles rapidly release myoglobin, leading to elevated serum and urinary myoglobin concentrations. Levels peak at $8-12$ hours, but typically normalise in serum within 24 hours. ${ }^{1}$ This was also found in our patient.

- A urine dipstick will indicate 'blood' in the urine, indicating presence in this case of myoglobin (not haemoglobin). As such, a combination of myoglobinuria and elevated plasma CK levels confirms the diagnosis of rhabdomyolysis. ${ }^{1}$

- Electrolyte abnormalities such as hyperkalaemia, hyperphosphataemia and hypocalcaemia occur, but are not specific enough for diagnostic certainty.

Administration of the rhabdomyolysing agents/medication should be stopped immediately to prevent further skeletal muscle damage. ${ }^{6}$ Control of fluid equilibrium is vital to stabilise the circulation and serum potassium and to prevent acidosis. ${ }^{1,6}$ After the corticosteroid dose is reduced or discontinued, the steroid-induced muscle weakness decreases, although it can take weeks or months for full recovery. ${ }^{3}$

\section{Conclusion}

Acute corticosteroid-induced rhabdomyolysis is rare but potentially life-threatening. Early diagnosis is vital in minimising the secondary damage.

\section{References}

1. Parekh R. Emergency Medicine Practice. Rhabdomyolysis: Advances in diagnosis and treatment. EB Medicine.net, 2012. http://www.ebmedicine.net/store. php?paction=showProduct\&pid=258 (accessed 1 September 2012).

2. Williams TJ, O'Hehir RE, Czarny D, Horne M, Bowes G. Acute myopathy in severe asthma treated with intravenously administered corticosteroids. Am Rev Respir Dis 1988;137:460-463.

3. Lim SS. Medscape Reference: Drugs, Diseases and Procedures. Corticosteroidinduced myopathy, 2010. http://emedicine.medscape.com/article/313842-overview (accessed 1 September 2012).

4. Takahashi N, Shimada T, Tanabe K, et al. Steroid-induced crisis and rhabdomyolysis in a patient with pheochromocytoma: A case report and review. Int J Cardiol 2011;146:e41-e45. [http://dx.doi.org/10.1016/j.ijcard.2008.12.183]

5. Hanson P, Dive A, Brucher J, Bisteau M, Dangoisse M, Deltome T. Acute corticosteroid myopathy in intensive care patients. Muscle Nerve 1997;20:1371-1380.

6. Hohenegger M. Drug induced rhabdomyolysis. Curr Opin Pharmacol 2012;12:1-5. [http://dx.doi.org/10.1016/j.coph.2012.04.002]

Table 1. Main differences between chronic and acute myopathy

\begin{tabular}{lll}
\hline & Chronic myopathy & Acute myopathy \\
\hline Muscle weakness & Proximal muscles & Proximal and distal muscles \\
CK levels & Normal/slightly elevated & Significantly higher, possible rhabdomyolysis \\
Muscle biopsy & Type IIB fibre atrophy & Focal and diffuse necrosis
\end{tabular}

